\title{
RHOA mutations and CLDN18-ARHGAP fusions in intestinal-type adenocarcinoma with anastomosing glands of the stomach
}

\author{
Taiki Hashimoto $\mathbb{1}^{1} \cdot$ Reiko Ogawa $^{2} \cdot$ Tzu-Yin Tang $^{1,3} \cdot$ Hiroshi Yoshida ${ }^{1} \cdot$ Hirokazu Taniguchi ${ }^{1} \cdot$ Hitoshi Katai $^{4} \cdot$ \\ Ichiro Oda ${ }^{5} \cdot$ Shigeki Sekine ${ }^{1,2}$
}

Received: 26 September 2018 / Revised: 29 October 2018 / Accepted: 30 October 2018 / Published online: 13 November 2018

(c) United States \& Canadian Academy of Pathology 2018

\begin{abstract}
A subtype of intestinal-type adenocarcinoma of the stomach, characterized by low-grade cytological atypia and anastomosing glands, has been described in several reports under different names. One of the remarkable features of these lesions, herein referred to as intestinal-type adenocarcinoma with anastomosing glands, is the frequent association of poorly differentiated adenocarcinoma components. Here we analyzed 44 intestinal-type adenocarcinomas with anastomosing glands focusing on the molecular abnormalities that are common in diffuse-type gastric cancers. Next-generation sequencing identified $R H O A$ and CDH1 mutations in $22(50 \%)$ and one lesion (2\%), respectively. Reverse transcription-PCR detected CLDN18-ARHGAP fusions in three lesions (7\%). Immunohistochemically, none of the lesions showed abnormal p53 expression patterns whereas focal and diffuse loss of ARID1A was observed in four and one lesion, respectively. Examination of 37 lesions of dysplasia and 26 usualtype intramucosal adenocarcinomas identified one RHOA mutation in adenocarcinoma and no CLDN18-ARHGAP fusions, indicating that these genetic alterations are highly specific to intestinal-type adenocarcinomas with anastomosing glands among differentiated-type intramucosal neoplasms. The present study showed that intestinal-type adenocarcinoma with anastomosing glands represents a genetically distinct group of tumors with the frequent presence of RHOA mutations and CLDN18-ARHGAP fusions, which are thought to be specific to diffuse-type gastric cancers.
\end{abstract}

\section{Introduction}

A group of gastric adenocarcinomas, characterized by irregularly fused/anastomosing glands and low-grade cytological

Electronic supplementary material The online version of this article (https://doi.org/10.1038/s41379-018-0181-9) contains supplementary material, which is available to authorized users.

Shigeki Sekine

ssekine@ncc.go.jp

1 Division of Pathology and Clinical Laboratories, National Cancer Center Hospital, Tokyo, Japan

2 Division of Molecular Pathology, National Cancer Center Research Institute, Tokyo, Japan

3 Department of Pathology, MacKay Memorial Hospital, Taipei, Taiwan

4 Division of Gastric Surgery, National Cancer Center Hospital, Tokyo, Japan

5 Endoscopy Division, National Cancer Center Hospital, Tokyo, Japan atypia, has been reported under different names, including well-differentiated adenocarcinoma mimicking completetype intestinal metaplasia [1], very well-differentiated gastric carcinoma of intestinal-type [2], and "crawling-type" adenocarcinoma [3, 4]. Despite some differences in definitions used in the respective studies, the previous reports described largely overlapping clinicopathological features, supporting that they represent a distinct subtype. These lesions are predominantly located in the middle third of the stomach [1-3] and endoscopically appear as ill-demarcated depressed lesions [2,3]. Most of them are confined to the mucosal layer [2-4] and often show features of completetype intestinal metaplasia $[1,3,4]$. The common association of a poorly differentiated adenocarcinoma component is another remarkable feature [2, 3]. Importantly, the biopsy diagnosis of these lesions is often challenging owing to the minimal cytological atypia [1-4].

Some studies named these lesions to reflect their primarily differentiated morphology and subtle cytological atypia as described above [1, 2]. However, irregularly interconnected glands and the association of poorly differentiated adenocarcinoma components are rather characteristic in these 
lesions $[2,3]$. Based on our experience in diagnostic situations, we believe that the architectural abnormality, particularly prominent anastomosing glands, is the defining morphological feature of this group of lesions. The term, "crawling-type" adenocarcinoma reflects this feature in part $[3,4]$, but is not quite straightforward. Therefore, we apply a descriptive term, intestinal-type adenocarcinoma with anastomosing glands, to represent these lesions in the present study.

Although several studies have characterized the morphological and immunohistochemical features of intestinal-type adenocarcinomas with anastomosing glands [1-4], their molecular backgrounds remain poorly understood. Woo et al. [4] conducted a detailed histology-based analysis and showed that intestinal-type adenocarcinomas with anastomosing glands, referred to as adenocarcinomas of crawling-type in this study, lack many of the molecular abnormalities frequently observed in intestinal-type adenocarcinomas, including HER2 overexpression, association of Epstein-Barr virus, mismatch repair deficiency, and loss of PTEN. Moreover, overexpression of p53 and MET was significantly less common compared to usual-type adenocarcinomas. Considering these findings and the fact that intestinaltype adenocarcinomas with anastomosing glands are frequently associated with poorly differentiated adenocarcinomas, these lesions may be more closely related to diffuse-type gastric cancers in terms of molecular features. The present study analyzed a series of intestinaltype adenocarcinomas with anastomosing glands focusing on molecular abnormalities that are common in diffuse-type adenocarcinomas and identified frequent RHOA mutations and CLDN18-ARHGAP fusions in these lesions.

\section{Materials and methods}

\section{Samples}

This study was approved by the Ethics Committee of the National Cancer Center, Tokyo, Japan. All tissue samples were obtained by endoscopic or surgical resection at the National Cancer Center Hospital, Tokyo, Japan. In the present study, we analyzed 44 intestinal-type adenocarcinomas with anastomosing glands, 37 lesions of dysplasia, and 26 differentiated-type intramucosal adenocarcinomas. We retrieved intestinal-type adenocarcinomas with anastomosing glands diagnosed between January 2012 and April 2018 and two pathologists (TH and SS) confirmed the diagnosis. Intestinal-type adenocarcinoma with anastomosing glands was defined as intramucosal intestinal-type adenocarcinoma with low-grade cytological atypia and frequent anastomosing glands. The lesions may resemble complete-type intestinal metaplasia in terms of the presence of absorptive, goblet, and/or Paneth cells. The lesions may be associated with components of other histological subtypes, focal areas of high-grade dysplasia, or areas of invasion into the submucosal or deeper layers. Diagnosis of dysplasia and intramucosal adenocarcinoma was made based on the previously published criteria [5].

\section{DNA and RNA extraction}

Deparaffinized, $10 \mu \mathrm{m}$-thick sections from each paraffin block were microdissected under a microscope using sterilized toothpicks in order to enrich for tumor content. The microdissected samples were subjected to DNA and RNA extraction using the QIAamp DNA FFPE Tissue Kit (Qiagen, Hilden, Germany) and RNeasy FFPE Kit (Qiagen), respectively. In three intestinal-type adenocarcinomas with anastomosing glands associated with discrete areas of poorly differentiated adenocarcinoma (IAAG22, 32 and 39), the respective components were separately dissected and subjected to DNA and RNA extraction.

\section{Next-generation and Sanger sequencing}

Next-generation sequencing targeting exons 2 and 3 of RHOA and the entire coding region of $\mathrm{CDH} 1$ was performed as described previously [6]. Next-generation sequencing libraries were prepared by two-step tailed PCR. The gene-specific primers used are listed in Supplementary Table 1. Sequencing was performed using MiSeq (Illumina, San Diego, CA, USA), according to the manufacturer's protocol. After excluding synonymous mutations and the known common single nucleotide polymorphisms based on dbSNP build 137, all mutations were verified by Sanger sequencing. In addition, the entire RHOA coding region was analyzed by Sanger sequencing in all intestinal-type adenocarcinomas with anastomosing glands using primers listed in Supplementary Table 2. For dysplasia lesions and intramucosal adenocarcinomas, exon 2 and 3 of $R H O A$, which harbor all the previously reported mutations in diffuse-type gastric cancers, were analyzed by Sanger sequencing.

\section{Reverse transcription (RT)-PCR}

Following RT reactions, cDNA sample quality was assessed by PCR amplification of a 220 base pair fragment of $A C T B$ [7]. PCRs targeting the fusion junctions of known $C L D N 18$ ARHGAP fusions, including CLDN18-ARHGAP26 and CLDN18-ARHGAP6 fusions [8-11], were performed using primers listed in Supplementary Table 3. All PCR products were verified by Sanger sequencing. 


\section{Immunohistochemistry}

Immunohistochemical analyses were performed on formalinfixed paraffin-embedded specimens. Antigen retrieval was performed by autoclaving the samples in $10 \mathrm{mM}$ citrate buffer (pH 6.0) for $10 \mathrm{~min}$. Anti-ARID1A (HPA005456; 1:2000; Sigma-Aldrich, St Louis, MO, USA) and anti-p53 (DO-7; 1:100; Dako, Glostrup, Denmark) antibodies were used as primary antibodies. An automated stainer (Dako) was used in accordance with the manufacturer's protocol. ChemMate EnVision (Dako) methods were used for detection. For p53 staining, diffuse and complete lack of staining were regarded as abnormal expression patterns. For ARID1A, areas devoid of expression, occupying $>10 \%$ of lesions, were regarded as significant.

\section{Statistical analysis}

Fisher's exact test and Welch's $t$ test were used to analyze categorical variables and continuous variables, respectively. $P$ values $<0.05$ were considered to indicate statistical significance.

\section{Results}

Forty-four intestinal-type adenocarcinomas with anastomosing glands were obtained from 26 male and 18 female patients aged 49-87 years. Eleven and 24 lesions were located in the upper and middle third of the stomach, respectively. They were $7-110 \mathrm{~mm}$ in size (median, $23 \mathrm{~mm}$ ). Histologically, all lesions contained variable proportions of tortuous and/or anastomosing tumor glands by definition (Fig. 1a-c). Tumor cells had basally located nuclei with minimal atypia and proliferating cells were confined to the lower portion of the neoplastic glands. Goblet cells were found at least focally in all the lesions and Paneth cells were also common (Fig. 1b). Distended glands, which are lined by flattened epithelium and often contain necrotic debris, were observed in 38 lesions (Fig. 1d). Twenty-four lesions showed focal foveolar epithelial differentiation (Fig. 1e). Eight lesions had focal areas of highgrade cytology. Discohesive cells were present in 22 lesions and invasion into the submucosal or deeper layer was observed in ten lesions (Fig. 1f).

To probe if intestinal-type adenocarcinomas with anastomosing glands share molecular features with diffuse-type cancer, we focused our analysis on genes that are frequently altered in diffuse-type adenocarcinomas. We reviewed previously reported large-scale genomic analyses of diffuse-type gastric cancers, and selected genetic alterations that were detected in more than $10 \%$ of cases, including $C D H 1, R H O A$, TP53, and ARIDIA mutations and CLDN18-ARHGAP fusions $[8,12,13]$.
Next-generation sequencing targeting $\mathrm{RHOA}$ and $\mathrm{CDHI}$ resulted in median total coverage per sample of 276,920 reads $($ range $=108,804-806,850$ ). Median coverage per amplicon across all samples was 5027.5 reads (range $=$ 110-67,602). The analysis identified missense RHOA mutations in 22 intestinal-type adenocarcinomas with anastomosing glands (50\%). Sanger sequencing confirmed all the mutations detected by next-generation sequencing and did not find any additional mutations (Fig. 2a, b and Supplementary Table 4). All the RHOA mutations were missense and were localized to the first two coding exons (exon 2 and 3). Comparison with previously reported results indicated that intestinal-type adenocarcinomas with anastomosing glands and diffuse-type gastric cancers show similar RHOA mutation patterns, including the presence of several hot spots $[8,12,13]$. A missense $C D H 1$ mutation (IAAG40; c.1757C > T, p.S586F) was identified in one lesion, concurrent with a RHOA mutation. RT-PCR detected the CLDN18-ARHGAP26 exon 12 fusion in two lesions and the CLDN18-ARHGAP26 exon 10 fusion in one lesion whereas the CLDN18-ARHGAP6 fusion was absent (Fig. 2c). All the CLDN18-ARHGAP26 fusions were mutually exclusive with RHOA mutations.

Given the presence of RHOA mutations and CLDN18ARHGAP26 fusions in a total of 25 intestinal-type adenocarcinomas with anastomosing glands (57\%), we sought to determine their specificity among differentiated-type intramucosal neoplasms. We analyzed 37 lesions of dysplasia and 26 intramucosal intestinal-type adenocarcinomas for the presence of RHOA mutations and CLDN18-ARHGAP fusions. The analysis identified a missense RHOA mutation in a case of intramucosal adenocarcinoma but none of the lesions had CLDN18-ARHGAP fusions. Thus, RHOA mutations and CLDN18-ARHGAP fusions are highly specific to intestinal-type adenocarcinomas with anastomosing glands among differentiated-type intramucosal neoplasms of the stomach. Notably, the RHOA mutation identified in differentiated-type adenocarcinoma (c.59G $>$ A, p.C20Y) was not observed in intestinal-type adenocarcinomas with anastomosing glands and has not previously been reported in diffuse-type gastric cancers (Fig. 2d) [8, 12, 13].

Because TP53 and ARIDIA mutations have been shown to correlate well with their protein expression $[14,15]$, we performed immunohistochemistry for p53 and ARID1A. All the lesions showed weak heterogeneous p53 staining, predominantly in the proliferative cells (Fig. 3a, b). ARID1A expression was retained in most lesions but one and four lesions exhibited diffuse and focal loss of expression, respectively (Fig. 3c, d). Areas of ARID1A loss did not show distinct histological features compared with the areas with retained ARID1A in the respective lesions.

Three intestinal-type adenocarcinomas with anastomosing glands were associated with discrete areas of poorly 
Fig. 1 Histology of intestinaltype adenocarcinoma with anastomosing glands. a Intestinal-type adenocarcinoma with anastomosing glands showing irregularly fused glands. The tumor is confined to the mucosal layer and shows surface maturation. b A lesion resembling complete-type intestinal metaplasia with welldeveloped goblet and Paneth cells. Tumor glands show horizontal extension and are irregularly interconnected. c A lesion with prominently tortuous glands. Goblet cells are rarely seen in this lesion. d Distended glands lined by flattened epithelium and containing necrotic debris. e A lesion showing foveolar epithelial differentiation characterized by apical mucin caps. f Discohesive cells intermingled with typical anastomosing glands of intestinal-type adenocarcinomas with anastomosing glands
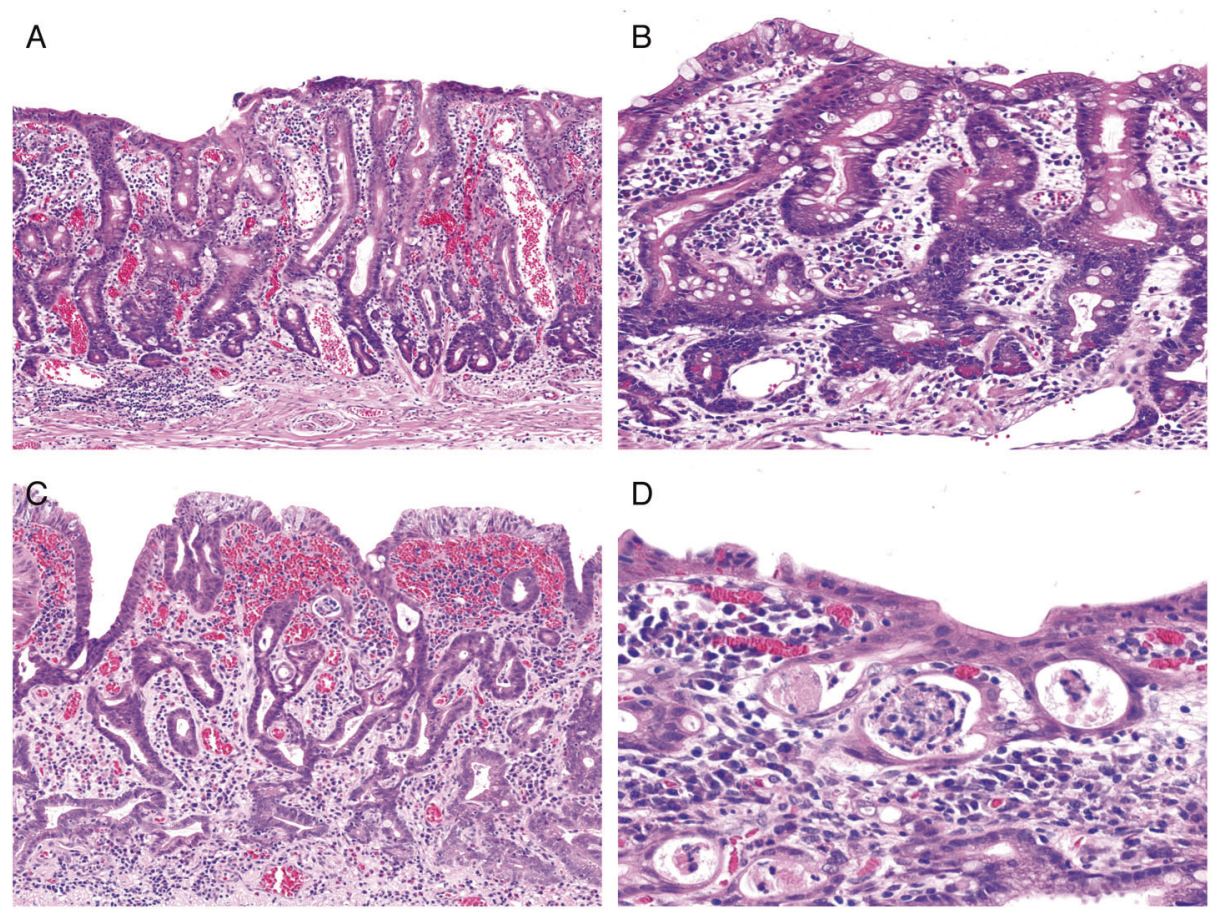

D
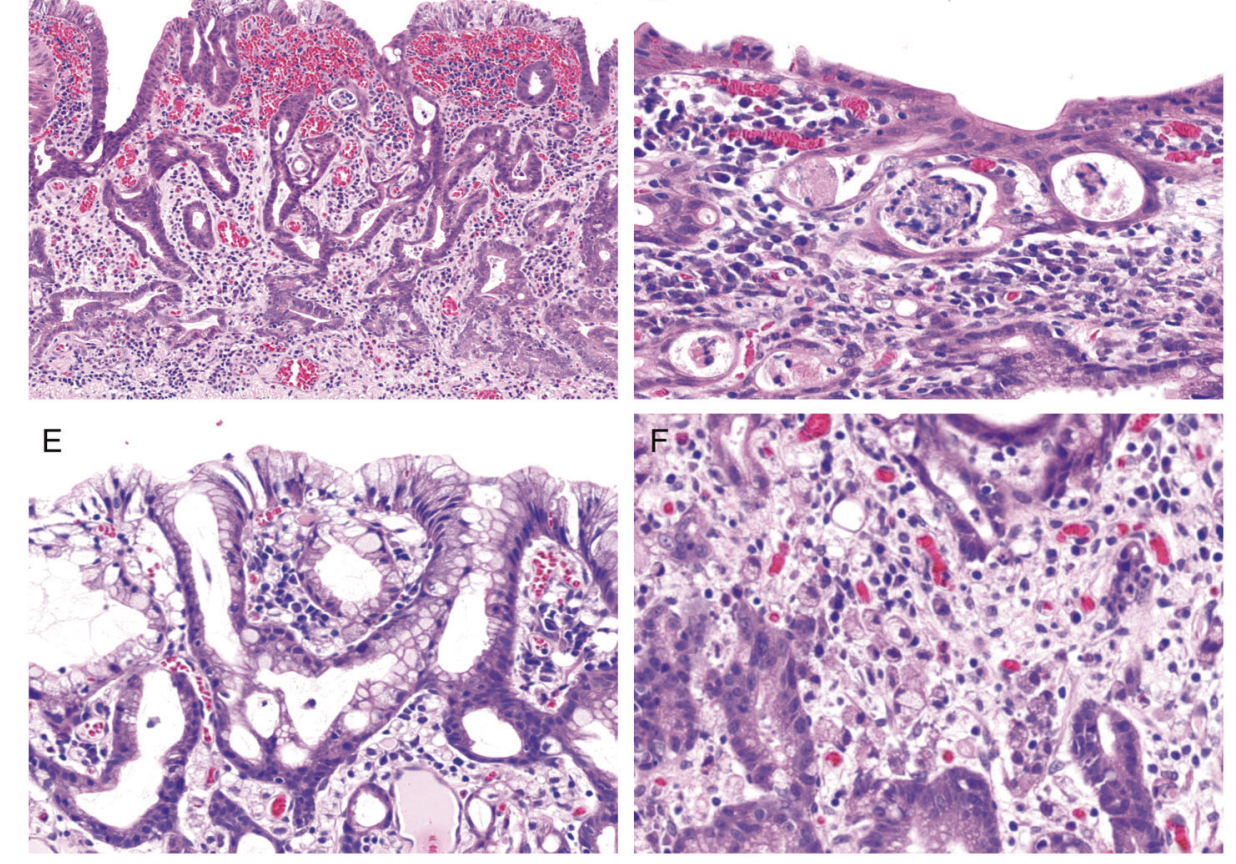

differentiated adenocarcinoma, and the respective components were separately analyzed for genetic alterations and protein expression as described above. In all three lesions, both components shared common RHOA mutations (Fig. 4). CDH1 mutation, abnormal p53 expression, and loss of ARID1A were not observed in any of the lesions.

Finally, we tested the clinicopathological significance of RHOA mutations in intestinal-type adenocarcinomas with anastomosing glands. The presence of RHOA mutations was not correlated with any of the clinicopathological parameters examined (Table 1).

\section{Discussion}

The findings of the present study showed that RHOA mutations are common in intestinal-type adenocarcinomas with anastomosing glands but very rare in usual-type intramucosal adenocarcinomas and dysplasia. Previous large-scale genomic analyses of gastric cancers showed
RHOA mutations to be virtually specific to diffuse-type adenocarcinomas with a prevalence of $14-25 \%$ [8, 12, 13]. It appears conflicting that intestinal-type adenocarcinomas with anastomosing glands have RHOA mutations with high frequency, even compared to that in diffuse-type gastric cancers, despite their intestinal-type morphology. Interestingly, a previous study reported that $73 \%$ of $\mathrm{RHOA}$ mutation-positive diffuse-type gastric cancers have minor components of tubular differentiation [16]. Indeed, all the three lesions associated with discrete areas of poorly differentiated adenocarcinoma had RHOA mutations in both components, indicating that RHOA mutations occur before transition from intestinal-type adenocarcinoma with anastomosing glands to poorly differentiated adenocarcinoma. There are two putative tumorigenic pathways of diffusetype gastric cancers recognized [17-19]. The first pathway represents the de novo development of poorly differentiated or signet ring cell adenocarcinoma, as well-characterized in hereditary diffuse gastric cancer [20-22]. The other pathway is transition from differentiated-type adenocarcinomas. 
A

RHOA mutation 50\%

CLDN18-ARHGAP fusion $7 \%$

CDH1 mutation $2 \%$

p53 abnormal expression $0 \%$

ARID1A loss $11 \%$

B

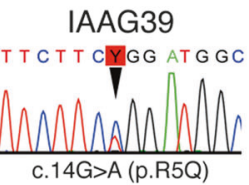

IAAG44

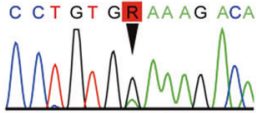
c.50G $>$ A (pG17E)

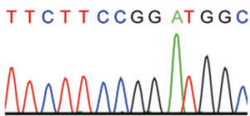

C CT GT G G AAAG ACA
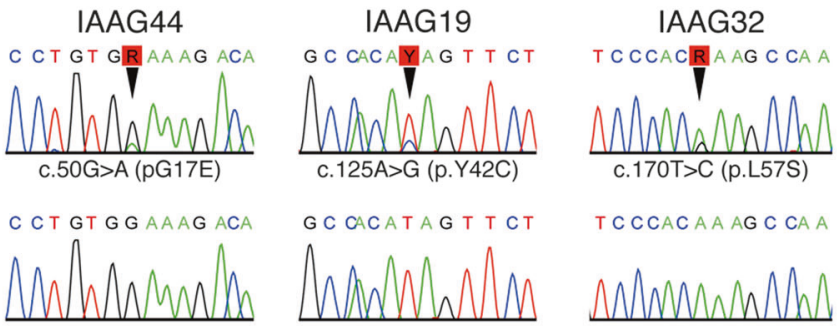

Wild-type

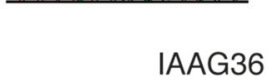

C

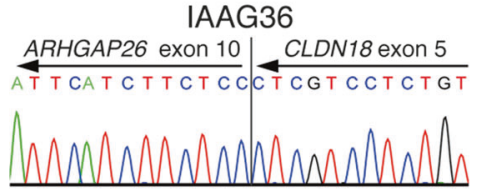

IAAG29

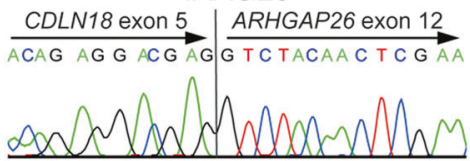

D Intestinal-type adenocarcinoma with anastomosing glands (22/44 cases, 50\%)

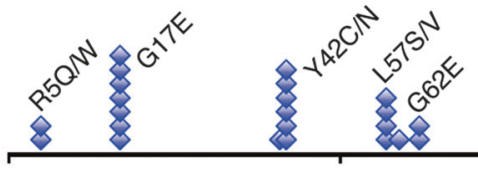

Usual-type adenocarcinoma/dysplasia (1/63 cases, $2 \%)$

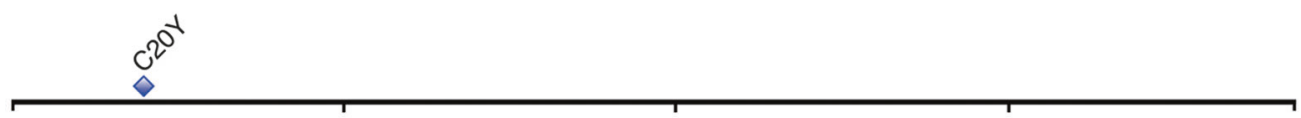

Diffuse-type adenocarcinoma* (38/215 cases, $18 \%)$

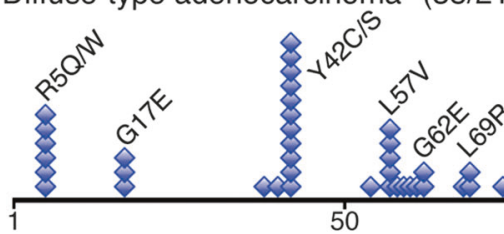

Fig. 2 Molecular characterization of intestinal-type adenocarcinoma with anastomosing glands. a Summary of sequencing, RT-PCR, and immunohistochemical studies. Half of the intestinal-type adenocarcinomas with anastomosing glands had RHOA mutations. CLDN18ARHGAP fusions were identified in three lesions and were mutually exclusive with RHOA mutations. b Sanger sequencing of RHOA. All

100

150

amino acids detected mutations were missense and affected the first two coding exons. c Sanger sequencing of CLDN18-ARHGAP26 fusion junctions. d Distribution of RHOA mutations. Notice the common mutation hot spots in intestinal-type adenocarcinomas with anastomosing glands and diffuse-type adenocarcinomas. *The mutation data of diffuse-type adenocarcinoma is adapted from previous reports $[8,12,13]$

that these RHOA mutations are gain-of-function mutations; however, a recent functional study demonstrated that they actually act as dominant-negatives and inhibit ROCK activity, resulting in enhanced cell survival and migration [23]. The presence of RHOA mutations was not correlated with any of the clinicopathological factors in intestinal-type adenocarcinomas with anastomosing glands. Thus, although the frequent presence of $R H O A$ mutations suggests their critical roles in the development of intestinal-type 
Fig. 3 Immunohistochemistry for p53 and ARID1A. a, b Weak nuclear expression of $\mathrm{p} 53$ is seen heterogeneously in proliferative cells, which are localized to the lower layer of the lesion. $\mathbf{c}, \mathbf{d}$ An intestinal-type adenocarcinoma with anastomosing glands with focal loss of ARID1A (c, right and d). There was no remarkable difference in the histology of areas with retained and loss of ARID1A
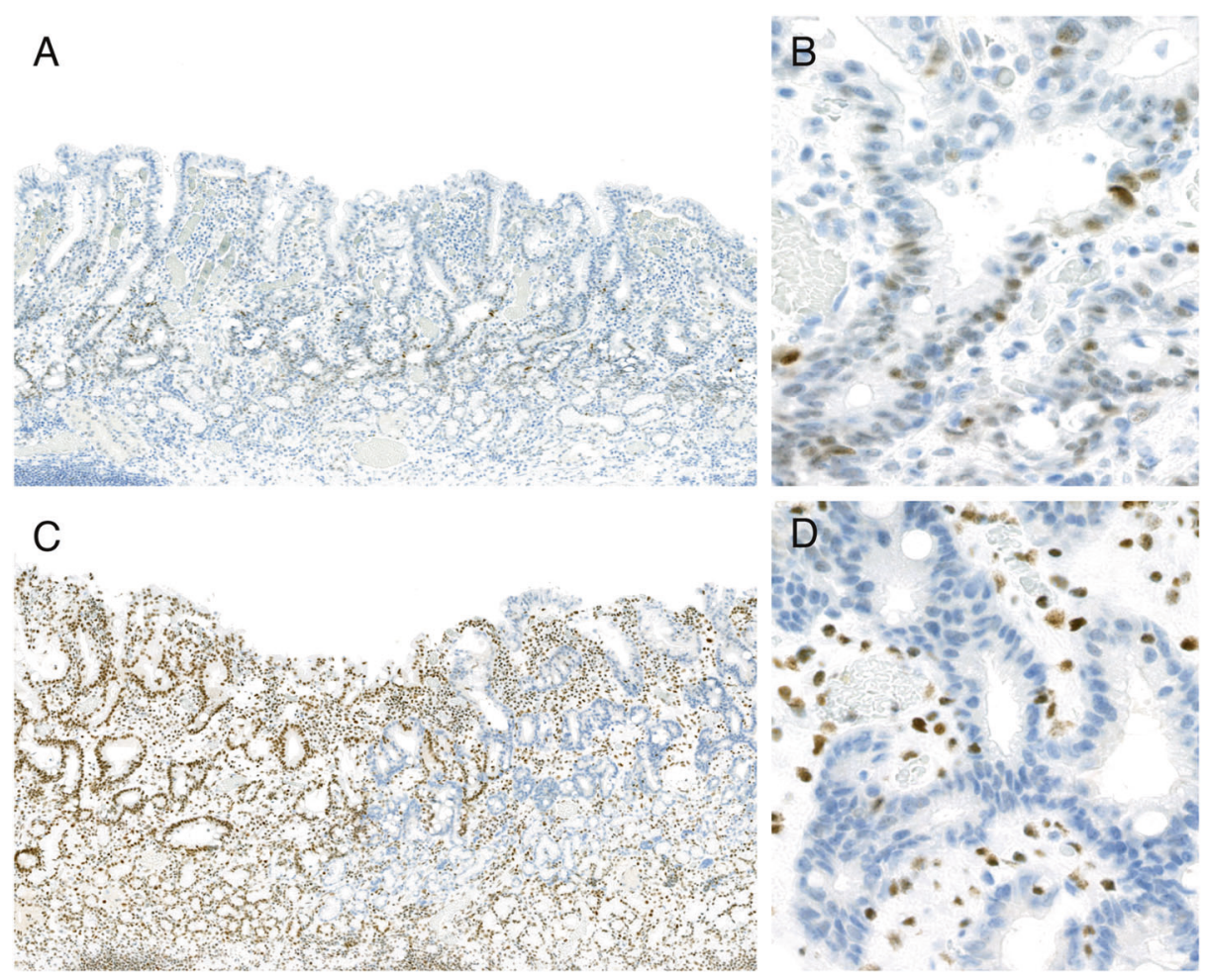

Fig. 4 An intestinal-type adenocarcinoma with anastomosing glands with a discrete area of poorly differentiated adenocarcinoma. a-c A lesion containing welldemarcated intestinal-type adenocarcinoma with anastomosing glands (a, left and b) and poorly differentiated adenocarcinoma components (a, right and c). d Sanger sequencing identified a common RHOA mutation in both components
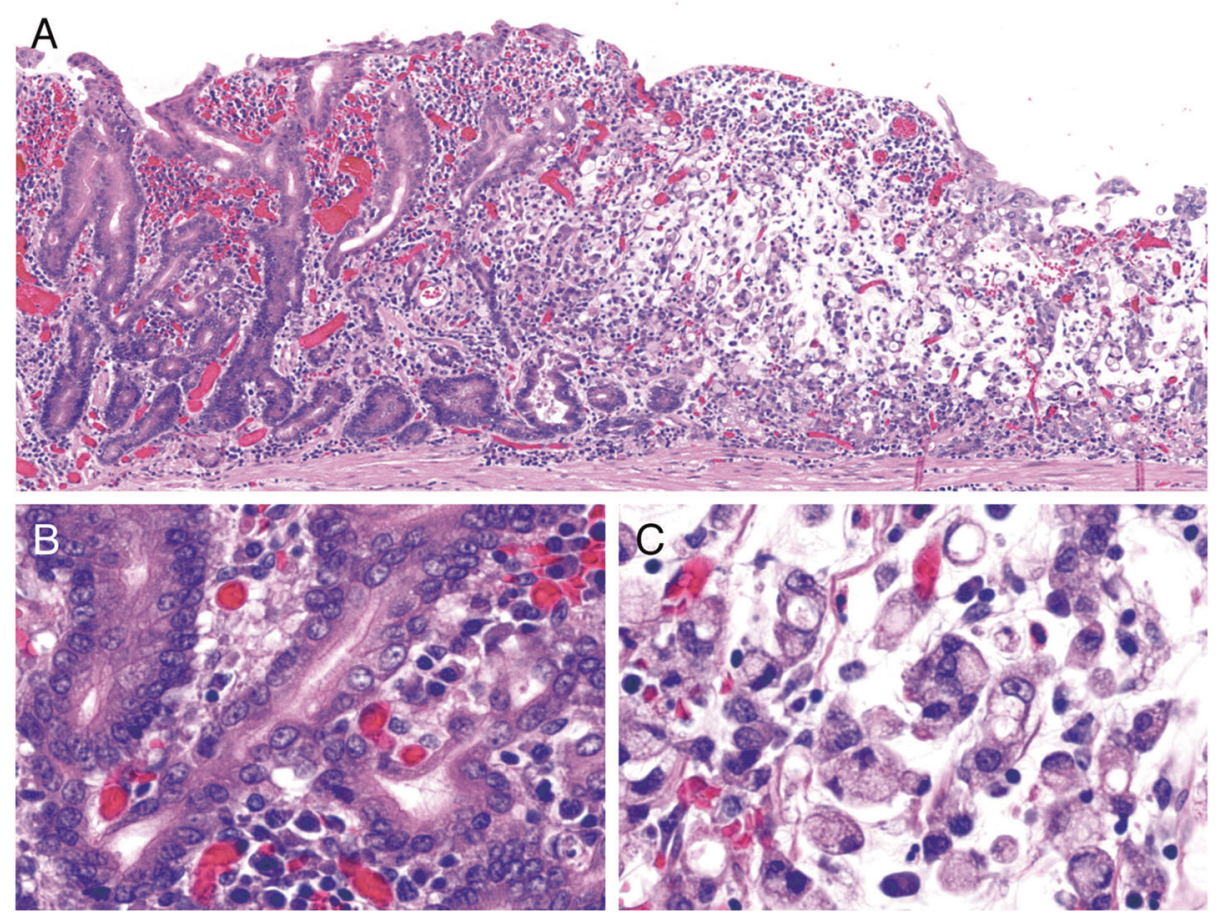

D

IAAG

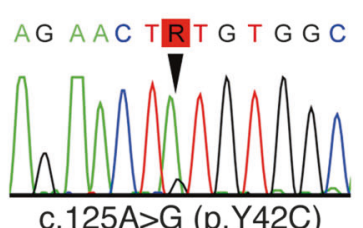

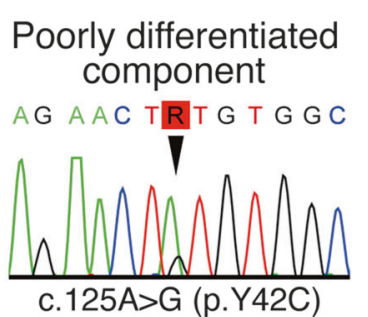

\section{Wild-type}

A G A A TA T G T G G C

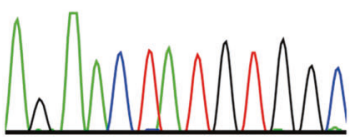


Table 1 Clinicopathological features of intestinal-type adenocarcinoma with anastomosing glands, depending on the RHOA mutation status

\begin{tabular}{llll}
\hline & \multicolumn{2}{l}{$R$ ROA mutation } & $P$ value \\
\cline { 2 - 3 } & $\begin{array}{l}\text { Positive }(\mathrm{n}= \\
\text { 22) }\end{array}$ & \\
& $22)$ & \\
\hline $\begin{array}{lll}\text { Age, year-old, median } \\
\text { (range) }\end{array}$ & $71.5(49-84)$ & $67.5(54-87)$ & 1.0 \\
Male/Female & $13 / 9$ & $13 / 9$ & 1.0 \\
Location, U/M/L & $6 / 13 / 3$ & $5 / 11 / 6$ & 0.61 \\
Size, mm, median & $32(13-110)$ & $17(7-109)$ & 0.13 \\
(range) $_{\text {Anastomosing glands }}{ }^{\mathrm{a}}$ & $0 / 4 / 12 / 6$ & $0 / 7 / 9 / 6$ & $1.0^{\mathrm{b}}$ \\
Distended glands & $1 / 21 / 0 / 0$ & $5 / 16 / 1 / 0$ & $0.19^{\mathrm{b}}$ \\
Discohesive cells $^{\mathrm{a}}$ & $8 / 9 / 5 / 0$ & $14 / 4 / 2 / 2$ & $0.13^{\mathrm{b}}$ \\
Microabscess $^{\mathrm{a}}$ & $8 / 13 / 1 / 0$ & $5 / 16 / 1 / 0$ & $0.51^{\mathrm{b}}$ \\
Foveolar epithelium $^{\mathrm{a}}$ & $9 / 13 / 0 / 0$ & $11 / 10 / 1 / 0$ & $0.76^{\mathrm{b}}$ \\
Paneth cells $^{\mathrm{a}}$ & $8 / 13 / 1 / 0$ & $2 / 16 / 4 / 0$ & $0.069^{\mathrm{b}}$ \\
Goblet cells $^{\mathrm{a}}$ & $0 / 5 / 4 / 13$ & $0 / 6 / 6 / 10$ & $1.0^{\mathrm{b}}$ \\
High-grade cytology $^{\mathrm{a}}$ & $19 / 3 / 0 / 0$ & $17 / 2 / 3 / 0$ & $0.70^{\mathrm{b}}$ \\
\hline
\end{tabular}

$U$ upper third of the stomach, $M$ middle third of the stomach, $L$ lower third of the stomach

${ }^{\mathrm{a}} 0 \% / 1-25 \% / 26-50 \% / 51-100 \%$

${ }^{b}<1 \%$ vs. $\geq 1 \%$

adenocarcinomas with anastomosing glands, they are of limited value in the subclassification of these lesions.

The identification of CLDN18-ARHGAP fusions in three intestinal-type adenocarcinomas with anastomosing glands is another remarkable finding of the present study. CLDN18-ARHGAP fusions have also been reported as diffuse-type gastric cancer-specific genetic alterations and are mutually exclusive with RHOA mutations [8-11]. Remarkably, CLDN18-ARHGAP fusion products inhibit RHOA activity [9], and could compensate for the knockdown of RHOA mutants in a cell viability assay [23]. Thus, both RHOA mutations and CLDN18-ARHGAP fusions similarly result in inhibition of RHOA. RHOA coordinates epithelial morphogenesis through the regulation of various cellular functions, including substrate adhesion, migration, cell-cell adhesion, and proliferation [9, 23-25]. Thus, RHOA mutations and CLDN18-ARHGAP fusions may directly contribute to the characteristic morphology of intestinal-type adenocarcinomas with anastomosing glands.

CDH1 encodes a cell adhesion molecule, E-cadherin, which plays a central role in the formation of adherens junction in epithelial cells [26]. Inactivation of $\mathrm{CDHI}$ is common in sporadic and hereditary diffuse-type gastric cancers and directly compromises the proper cell-cell adhesion, consistent with their discohesive morphology [27-29]. Although intestinal-type adenocarcinomas with anastomosing glands often contain discohesive cells, $\mathrm{CDH}$ mutations were absent in all but one case of intestinal-type adenocarcinoma with anastomosing glands, thus genetically distinguishing them from diffuse-type gastric cancers.

None of the intestinal-type adenocarcinomas with anastomosing glands exhibited abnormal p53 expression, including overexpression and loss of expression, suggesting the lack of TP53 mutations in these lesions. This observation seems reasonable considering that abnormal expression of p53 is rare in lesions with low-grade dysplasia [30, 31]. Also, our result is in agreement with a previous study reporting the low prevalence of abnormal p53 expression in "crawling-type" adenocarcinomas [3, 4]. Focal and diffuse loss of ARID1A was observed in four and one lesion, respectively. The low prevalence and focality of ARID1A loss indicate that ARIDIA inactivation is not generally involved in the development of intestinal-type adenocarcinomas with anastomosing glands, but may play a role in progression to more advanced lesions.

The present study demonstrated the frequent presence of RHOA mutations and CLDN18-ARHGAP fusions, which have been regarded as specific to diffuse-type gastric cancer, in intestinal-type adenocarcinomas with anastomosing glands. This indicates that intestinal-type adenocarcinomas with anastomosing glands are a morphologically as well as genetically distinct subtype of gastric adenocarcinoma. The common presence of genetic alterations leading to inhibition of RHOA activity might underlie their unique morphological features and the frequent transition to poorly differentiated adenocarcinoma.

Acknowledgements We thank Ms. Sachiko Miura, Ms. Toshiko Sakaguchi, and Ms. Chizu Kina for their skillful technical assistance.

\section{Compliance with ethical standards}

Conflict of interest The authors declare that they have no conflict of interest.

\section{References}

1. Endoh Y, Tamura G, Motoyama T, Ajioka Y, Watanabe H. Welldifferentiated adenocarcinoma mimicking complete-type intestinal metaplasia in the stomach. Hum Pathol. 1999;30:826-32.

2. Ushiku T, Arnason T, Ban S, Hishima T, Shimizu M, Fukayama $\mathrm{M}$, et al. Very well-differentiated gastric carcinoma of intestinal type: analysis of diagnostic criteria. Mod Pathol. 2013;26:1620-31.

3. Okamoto N, Kawachi H, Yoshida T, Kitagaki K, Sekine M, Kojima K, et al. "Crawling-type" adenocarcinoma of the stomach: a distinct entity preceding poorly differentiated adenocarcinoma. Gastric Cancer. 2013;16:220-32.

4. Woo HY, Bae YS, Kim JH, Lee SK, Lee YC, Cheong JH, et al. Distinct expression profile of key molecules in crawling-type early gastric carcinoma. Gastric Cancer. 2017;20:612-9.

5. Sakurai U, Lauwers GY, Vieth M, Sawabe M, Arai T, Yoshida $\mathrm{T}$, et al. Gastric high-grade dysplasia can be associated with submucosal invasion: evaluation of its prevalence in a series of 
121 endoscopically resected specimens. Am J Surg Pathol. 2014;38:1545-50.

6. Sekine S, Mori T, Ogawa R, Tanaka M, Yoshida H, Taniguchi $\mathrm{H}$, et al. Mismatch repair deficiency commonly precedes adenoma formation in Lynch Syndrome-Associated colorectal tumorigenesis. Mod Pathol. 2017;30:1144-51.

7. Sekine S, Yamashita S, Tanabe T, Hashimoto T, Yoshida H, Taniguchi H, et al. Frequent PTPRK-RSPO3 fusions and RNF43 mutations in colorectal traditional serrated adenoma. J Pathol. 2016;239:133-8.

8. Cancer Genome Atlas Research Network. Comprehensive molecular characterization of gastric adenocarcinoma. Nature. 2014;513:202-9.

9. Yao F, Kausalya JP, Sia YY, Teo AS, Lee WH, Ong AG, et al. Recurrent Fusion Genes in Gastric Cancer: CLDN18-ARHGAP26 Induces Loss of Epithelial Integrity. Cell Rep. 2015;12:272-85.

10. Shu Y, Zhang W, Hou Q, Zhao L, Zhang S, Zhou J, et al. Prognostic significance of frequent CLDN18-ARHGAP26/6 fusion in gastric signet-ring cell cancer. Nat Commun. 2018;9:2447.

11. Tanaka A, Ishikawa $S$, Ushiku T, Yamazawa S, Katoh H, Hayashi A, et al. Frequent CLDN18-ARHGAP fusion in highly metastatic diffuse-type gastric cancer with relatively early onset. Oncotarget. 2018;9:29336-50.

12. Kakiuchi M, Nishizawa T, Ueda H, Gotoh K, Tanaka A, Hayashi A, et al. Recurrent gain-of-function mutations of RHOA in diffuse-type gastric carcinoma. Nat Genet. 2014;46:583-7.

13. Wang K, Yuen ST, Xu J, Lee SP, Yan HH, Shi ST, et al. Wholegenome sequencing and comprehensive molecular profiling identify new driver mutations in gastric cancer. Nat Genet. 2014;46:573-82.

14. Finlay CA, Hinds PW, Tan TH, Eliyahu D, Oren M, Levine AJ. Activating mutations for transformation by 553 produce a gene product that forms an hsc70-p53 complex with an altered half-life. Mol Cell Biol. 1988;8:531-9.

15. Wang K, Kan J, Yuen ST, Shi ST, Chu KM, Law S, et al. Exome sequencing identifies frequent mutation of ARID1A in molecular subtypes of gastric cancer. Nat Genet. 2011;43:1219-23.

16. Ushiku T, Ishikawa S, Kakiuchi M, Tanaka A, Katoh H, Aburatani $\mathrm{H}$, et al. RHOA mutation in diffuse-type gastric cancer: a comparative clinicopathology analysis of 87 cases. Gastric Cancer. 2016;19:403-11.

17. Saito A, Shimoda T, Nakanishi Y, Ochiai A, Toda G. Histologic heterogeneity and mucin phenotypic expression in early gastric cancer. Pathol Int. 2001;51:165-71.

18. Natsagdorj L, Sugihara H, Bamba M, Hattori T. Intratumoural heterogeneity of intestinal expression reflects environmental induction and progression-related loss of induction in undifferentiated-type gastric carcinomas. Histopathology . 2008;53:685-97.

19. Ikarashi S, Nishikura K, Ajioka Y, Aoyagi Y. Re-evaluation of phenotypic expression in undifferentiated-type early gastric adenocarcinomas using mucin core protein and CDX2. Gastric Cancer. 2013;16:208-19.

20. Huntsman DG, Carneiro F, Lewis FR, MacLeod PM, Hayashi A, Monaghan KG, et al. Early gastric cancer in young, asymptomatic carriers of germ-line E-cadherin mutations. $N$ Engl $\mathrm{J}$ Med. 2001;344:1904-9.

21. Carneiro F, Huntsman DG, Smyrk TC, Owen DA, Seruca R, Pharoah P, et al. Model of the early development of diffuse gastric cancer in E-cadherin mutation carriers and its implications for patient screening. J Pathol. 2004;203:681-7.

22. Barber ME, Save V, Carneiro F, Dwerryhouse S, Lao-Sirieix P, Hardwick RH, et al. Histopathological and molecular analysis of gastrectomy specimens from hereditary diffuse gastric cancer patients has implications for endoscopic surveillance of individuals at risk. J Pathol. 2008;216:286-94.

23. Nishizawa T, Nakano K, Harada A, Kakiuchi M, Funahashi SI, Suzuki M, et al. DGC-specific RHOA mutations maintained cancer cell survival and promoted cell migration via ROCK inactivation. Oncotarget. 2018;9:23198-207.

24. Parri M, Chiarugi P. Rac and Rho GTPases in cancer cell motility control. Cell Commun Signal. 2010;8:23.

25. Zhou X, Zheng Y. Cell type-specific signaling function of RhoA GTPase: lessons from mouse gene targeting. J Biol Chem. 2013;288:36179-88.

26. Takeichi M. Cadherin cell adhesion receptors as a morphogenetic regulator. Science. 1991;251:1451-5.

27. Becker KF, Atkinson MJ, Reich U, Becker I, Nekarda H, Siewert $\mathrm{JR}$, et al. E-cadherin gene mutations provide clues to diffuse type gastric carcinomas. Cancer Res. 1994;54:3845-52.

28. Guilford P, Hopkins J, Harraway J, McLeod M, McLeod N, Harawira $\mathrm{P}$, et al. E-cadherin germline mutations in familial gastric cancer. Nature. 1998;392:402-5.

29. Grady WM, Willis J, Guilford PJ, Dunbier AK, Toro TT, Lynch $\mathrm{H}$, et al. Methylation of the $\mathrm{CDH} 1$ promoter as the second genetic hit in hereditary diffuse gastric cancer. Nat Genet. 2000;26:16-7.

30. Rugge M, Shiao YH, Correa P, Baffa R, DiMario F. Immunohistochemical evidence of $\mathrm{p} 53$ overexpression in gastric epithelial dysplasia. Cancer Epidemiol Biomark Prev. 1992;1:551-4.

31. Miracco C, Spina D, Vindigni C, Filipe MI, Tosi P. Cell proliferation patterns and p53 expression in gastric dysplasia. Int $\mathrm{J}$ Cancer. 1995;62:149-54. 